\section{Material particulado y gases contaminantes en la comuna de El Bosque ¿cuánto influyen en la cantidad de consultas por enfermedades respiratorias?}

\author{
GLORIA HENRÍQUEZ ${ }^{1, a}$, CLAUDIO URREA ${ }^{2, b}$
}

\section{Association between air pollution and emergency consultations for respiratory diseases}

Background: Air pollution has a direct influence on health. Aim: To determine the association between particulate matter and contaminant gas concentrations in the environment with the number of consultations for respiratory diseases in emergency rooms in Metropolitan Santiago, Chile. Material and Methods: During five years, the daily number emergency consultations for respiratory diseases and the daily concentrations of particulate matter and contaminant gases in a community of Santiago, were recorded. The degree of change of these variables during summer and winter was determined. Their correlation coefficients with a 0 to 100 days gap, were calculated. Results: During winter, there was a higher number of consultations and higher pollution levels, except for $\mathrm{O}_{3}$, which increased in summer. There were positive correlations between the concentrations of different pollutants (mainly 2.5 and $10 \mu \mathrm{m}$ particulate matter, $\mathrm{CO}$ and $\mathrm{NO}_{2}$ ). There was a negative association between consultations for respiratory diseases and $\mathrm{O}_{3}$ concentrations, an almost negligible association with $\mathrm{SO}_{2}$ and variable positive and significant associations with the concentration of other pollutants, with variations according to the time gap. Conclusions: Pollution and respiratory diseases increase during winter. There are variable associations between pollutant concentrations and the number of consultations for respiratory diseases.

(Rev Med Chile 2017; 145: 1371-1377)

Key words: Air Pollution; Gases; Particulate Matter; Respiration Disorders; Respiratory Tract Diseases.

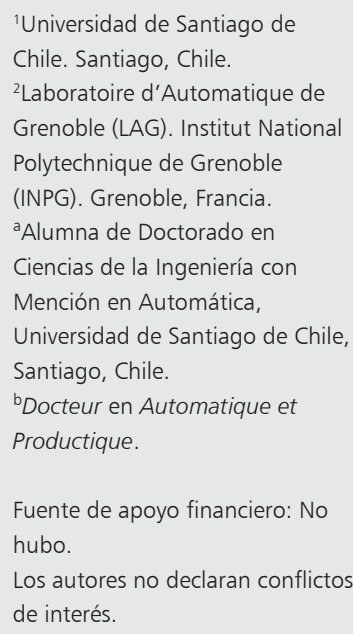

Fuente de apoyo financiero: No hubo.

Los autores no declaran conflictos de interés.

Recibido el 20 de enero de 2017, aceptado el 19 de noviembre de 2017.

Correspondencia a:

Gloria Henríquez

Universidad de Santiago de Chile Av. Ecuador 3519, Estación

Central, Santiago, Chile.

gloria.henriquez@usach.cl

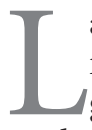

a contaminación del aire es un problema mundial que afecta principalmente a las grandes ciudades en países desarrollados y no desarrollados, con consecuencias para la salud de la población ${ }^{1}$. Debido a esto se han realizado múltiples estudios con el fin de establecer el daño causado, tanto por el material particulado como por los gases contaminantes, siendo las relaciones más conocidas el aumento de la mortalidad ${ }^{1,2}$ por exposición a corto plazo ${ }^{3}$ y a largo plazo $^{4}$, la asociación con enfermedades cardiovasculares, incluidas la hipertensión $\operatorname{arterial}^{5}$ y la menor sobrevida de personas con infarto de miocardio ${ }^{6}$, alteraciones cardiopulmonares ${ }^{7}$, problemas respiratorios como disminución de la función pulmonar ${ }^{8} \mathrm{y}$ aparición de asma en niños ${ }^{9}$, etc. Menos conocidas son las 
asociaciones con condiciones como disminución de la función renal ${ }^{10} \mathrm{y}$ alteraciones cognitivas ${ }^{11}$ en adultos mayores.

En Chile se comenzó la pesquisa diaria de material particulado de $10 \mu \mathrm{m}\left(\mathrm{MP}_{10}\right)$ desde $1989^{2}$ $\mathrm{y}$, actualmente, se monitorean aquellos contaminantes que originan situaciones de emergencia ambiental, agregándose al $\mathrm{MP}_{10}$ las mediciones de material particulado de $2,5 \mu \mathrm{m}\left(\mathrm{MP}_{2,5}\right)$, monóxido de carbono (CO), dióxido de nitrógeno $\left(\mathrm{NO}_{2}\right)$, ozono $\left(\mathrm{O}_{3}\right)$ y dióxido de azufre $\left(\mathrm{SO}_{2}\right)$. Las estaciones de monitoreo de calidad del aire se encuentran a lo largo de todo el país, siendo Santiago la ciudad con más antecedentes históricos ${ }^{12}$. Los estudios dirigidos a la relación contaminación -mortalidad han mostrado resultados similares a otros países $^{2} y$, progresivamente, se han ido investigado asociaciones con otras variables influyentes en la relación contaminación- salud, como factores climáticos $^{13,14} \mathrm{o}$ microorganismos patógenos ${ }^{15}$ e, incluso, estimando el efecto económico en la reducción de contaminantes ${ }^{16}$, entre otros.

El mayor problema de los estudios de contaminación versus salud radica en la imposibilidad de evaluar en forma aislada el daño que causa cada contaminante, incluso, algunos autores han realizado trabajos conducentes a determinar si el material particulado o los gases contaminantes son factores modificadores de efecto o causantes reales del daño que se les imputa ${ }^{17,18}$. Por lo anterior, en este trabajo se intenta establecer en qué proporción el material particulado $\left(\mathrm{MP}_{2,5} \mathrm{y} \mathrm{MP}_{10}\right)$ y los gases contaminantes $\left(\mathrm{CO}, \mathrm{NO}_{2}, \mathrm{O}_{3}\right.$ y $\mathrm{SO}_{2}$ ) influyen en la cantidad de atenciones médicas de urgencia a causa de enfermedades respiratorias, y determinar la existencia de desfase de tiempo que marque esta asociación. Los datos utilizados provienen de mediciones de contaminantes del aire y atenciones médicas realizadas en la comuna de El Bosque, ubicada en Santiago de Chile, en el período comprendido entre el 01 de enero de 2010 y el 31 de diciembre de 2014.

\section{Material y Método}

\section{Generalidades y características de los datos}

La comuna de El Bosque tiene una superficie de $14,2 \mathrm{~km}^{2}$ y está ubicada en la Región Metropolitana de Santiago de Chile. La población total proyectada para el año 2015 es de 193.915 habitantes, de los cuales, 20,69\% corresponde a niños de hasta 14 años y 9,52\% a adultos de 65 años o más. La población inscrita en el sistema público de salud es alrededor de $77 \%$, mientras que el resto lo está en el sistema privado, o no está en ninguno ${ }^{19}$.

Se ha seleccionado El Bosque para realizar el estudio porque cumple, en forma conjunta, con dos condiciones:

1. sus centros de atención primaria de salud (del sistema público) solamente atienden personas que viven en la misma comuna ${ }^{20}, \mathrm{y}$

2. su estación de monitoreo de calidad del aire tiene instalaciones que miden todos los contaminantes antes mencionados ${ }^{21}$.

El período de estudio es de 5 años, correspondiente a 1.826 días (desde el 01 de enero de 2010 hasta el 31 de diciembre de 2014) y los datos se agrupan en:

1. cantidad diaria de atenciones médicas de urgencia en los servicios de atención primaria de El Bosque (subdivididos en atenciones totales, por causa respiratoria y no respiratoria), obtenidos de la plataforma web del Departamento de Estadística e Información de Salud (DEIS) del Ministerio de Salud de Chile ${ }^{22}$, y

2. promedio diario de concentraciones de $\mathrm{MP}_{2.5}$, $\mathrm{MP}_{10}, \mathrm{CO}, \mathrm{NO}_{2}, \mathrm{O}_{3}$ y SO $\mathrm{SO}_{2}$ obtenidos de la página web del Sistema de Información Nacional de Calidad del Aire (SINCA) del Ministerio del Medio Ambiente de Chile ${ }^{21}$.

Los datos faltantes fueron imputados por medias condicionadas de datos agrupados ${ }^{23}$. El procedimiento se realizó calculando el promedio de las mediciones ocurridas en el mismo día y mes de los otros años registrados, es decir, si el dato faltante es del 17 de mayo de 2011, entonces se sustituyó por el promedio obtenido del 17 de mayo de los años 2010, 2012, 2013 y 2014. Con este procedimiento se intenta seguir la tendencia de cada época del año. El contaminante con más datos imputados fue el $\mathrm{SO}_{2}$, siendo menos de $3 \%$, mientras que las enfermedades respiratorias no requirieron imputación.

\section{Método}

En la zona estudiada, el invierno se caracteriza por temperaturas frías, asociadas a mala ventilación del aire con el consiguiente aumento en la concentración de contaminantes como $\mathrm{MP}_{2,5}$, 
$\mathrm{MP}_{10}, \mathrm{CO}, \mathrm{NO}_{2}$ y $\mathrm{SO}_{2}$, mientras que en verano se incrementa la temperatura $y$, aunque mejoran las condiciones de ventilación, esto permite una mayor concentración de $\mathrm{O}_{3}^{2,12}$.

Con la finalidad de determinar cómo se expresan las enfermedades respiratorias y los contaminantes en invierno y verano, se considera al primero como la estación con mayor exposición (por su concentración de contaminantes), definiéndose el invierno entre el 21 de junio y el 21 de septiembre, y el verano entre el 21 de diciembre y el 21 de marzo. Las atenciones de urgencia han sido calculadas para cada uno de esos períodos, subdivididas en "respiratorias" y "otra causa", obteniéndose la odds ratio y la significancia estadística por medio de $\chi^{2}$, mientras que los contaminantes se representaron según el promedio diario de concentraciones en cada período, y su significancia estadística obtenida por t-Student.

La relación entre contaminantes se ha revisado por medio de los coeficientes de correlación, calculados a partir de sus promedios diarios, y la prueba estadística utilizada ha sido t-Student.

La asociación entre atenciones diarias por enfermedades respiratorias y la concentración diaria de cada contaminante se ha analizado con el cálculo del coeficiente de correlación, con prueba estadística t-Student, inicialmente considerando ambas variables con ocurrencia en el mismo instante de tiempo $\left(\mathrm{t}_{0}\right) \mathrm{y}$, luego, manteniendo el contaminante sin modificación en el tiempo, mientras que las enfermedades respiratorias se fueron desfasando de 10 en 10 días hasta llegar a una diferencia de 100 días (el contaminante en tiempo inicial $t_{0}$ comparado con enfermedades respiratorias en los tiempos $\left.t_{10}, t_{20}, \ldots, t_{100}\right)$. Esto ha implicado la necesidad de completar el procedimiento utilizando datos de enfermedades respiratorias del año 2015, es decir, para el desfase de 100 días se tuvo que recurrir a las enfermedades cuantificadas hasta el 10 de abril de 2015.

\section{Resultados}

En los 5 años estudiados, los promedios diarios de concentraciones de $\mathrm{MP}_{2,5}$ y $\mathrm{MP}_{10}$ se mantuvieron por sobre las recomendaciones de la Organización Mundial de la Salud (promedio anual mayor a 10 y $20 \mu \mathrm{g} / \mathrm{m}^{3}$ y promedio en $24 \mathrm{~h}$ 25 y $50 \mu \mathrm{g} / \mathrm{m}^{3}$, respectivamente) ${ }^{1}$. La concentra- ción promedio diaria de $\mathrm{CO}$ en invierno fue más del triple que en verano, y la relación fue casi el doble en los casos de $\mathrm{MP}_{2,5}$ y NO$_{2}$. El MP ${ }_{10}$ y el $\mathrm{SO}_{2}$ no presentaron una diferencia tan marcada, mientras que el $\mathrm{O}_{3}$ fue 2,5 veces mayor en verano que en invierno. La desviación estándar fue similar entre ambas estaciones solamente en el caso del $\mathrm{O}_{3}$. Usando la prueba t-Student la significancia estadística, en todos los casos, dio un valor $\mathrm{p}<0,001$ para dos colas (Tabla 1 ).

El total de las atenciones de urgencia en los servicios de atención primaria, en el período 2010 2014 fue 750.549 , donde 309.023 correspondieron a patologías respiratorias y 441.526 se debieron a otra causa. Al analizar la cantidad de atenciones según las estaciones del año previamente definidas, se vio la predominancia de causa respiratoria en invierno y otras causas en verano (Tabla 2), siendo la odds ratio 2,2646 (IC 95\% 2,2337-2,2960) a favor de la relación invierno-enfermedades respiratorias y verano-otra causa y 0,4416 (IC 95\% 0,4355$0,4477)$ para la relación verano -enfermedades respiratorias e invierno- otra causa, con $\chi^{2}$ de un grado de libertad igual a $13.759,4947$, lo que produce un valor $\mathrm{p}<0,001$.

Los coeficientes de correlación más relevantes entre contaminantes se dieron en las relaciones $\mathrm{MP}_{2,5}-\mathrm{CO}, \mathrm{MP}_{2,5}-\mathrm{MP}_{10}$ y $\mathrm{MP}_{10}-\mathrm{NO}_{2}$ con valores de 0,81 o más. Le siguieron $\mathrm{MP}_{2,5}-\mathrm{NO}_{2}, \mathrm{NO}_{2}$ - $\mathrm{CO}$ y $\mathrm{MP}_{10}$ - $\mathrm{CO}$ con valores sobre 0,69 . $\mathrm{El} \mathrm{O}_{3}$ se asoció en forma negativa con todos los contaminantes, sobre todo con respecto al $\mathrm{CO}$ y $\mathrm{NO}_{2}$ (valores menores a 0,5 ). El SO fue el que presentó correlaciones con coeficientes más cercanos a cero (Tabla 3). La prueba estadística t-Student dio un valor $\mathrm{p}<0,001$ para dos colas en todos los casos.

Las mayores correlaciones entre enfermedades respiratorias y cada contaminante fueron las relacionadas con el $\mathrm{O}_{3}, \mathrm{NO}_{2}$ y CO. Le siguieron las del $\mathrm{MP}_{2,5}$ y $\mathrm{MP}_{10}$, mientras que el $\mathrm{SO}_{2}$ fue el que tuvo los valores más bajos del grupo (Tabla 4 ):

- La correlación más significativa $(-0,4834)$ se produjo al desfasar alrededor de 20 días las enfermedades respiratorias con respecto $\mathrm{al}_{3}$; debe destacarse que fue el único contaminante que entrega resultados negativos.

- El $\mathrm{NO}_{2}$ presentó dos peaks, el primero con alrededor de 20 días de diferencia respecto a las enfermedades respiratorias y el segundo a los 60 días, con coeficientes de 0,4669 y 0,3871, respectivamente. 
Tabla 1. Promedio diario de concentraciones de contaminantes y resultados comparativos entre estaciones invierno-verano del período 2010-2014

\begin{tabular}{|c|c|c|c|c|c|c|}
\hline \multirow[t]{2}{*}{ Ítem } & \multicolumn{6}{|c|}{ Contaminantes } \\
\hline & $\mathbf{M P}_{2,5}$ & $\mathbf{M P}_{10}$ & CO & $\mathrm{NO}_{2}$ & $\mathbf{O}_{3}$ & $\mathrm{SO}_{2}$ \\
\hline $\mathrm{P}_{\text {Total período }}$ & 29,1389 & 72,1240 & 0,9389 & 24,3611 & 13,1786 & 1,6465 \\
\hline$P_{\text {Inviernos }}$ & 38,0300 & 77,9686 & 1,4506 & 29,8032 & 7,5801 & 1,8408 \\
\hline$P_{\text {Veranos }}$ & 20,0880 & 63,3500 & 0,4312 & 16,5465 & 19,0482 & 1,3451 \\
\hline Inviernos/veranos & 1,8932 & 1,2308 & 3,3641 & 1,8012 & 0,3979 & 1,3685 \\
\hline D.S..$_{\text {Total período }}$ & 17,3352 & 30,3257 & 0,6922 & 11,0912 & 6,5912 & 0,8778 \\
\hline D.S. Inviernos $_{\text {. }}$ & 20,3080 & 33,6506 & 0,7346 & 10,2247 & 4,2382 & 1,0558 \\
\hline D.S. Veranos & 8,2171 & 18,5311 & 0,1746 & 5,4648 & 4,4347 & 0,5438 \\
\hline V.C. & 241,6741 & 741,7882 & 0,2876 & 67,5738 & 18,8062 & 0,7093 \\
\hline t-Student & 17,4166 & 8,0998 & 28,6877 & 24,3364 & 39,9069 & 8,8825 \\
\hline Valor $\mathrm{p}$ & $<0,001$ & $<0,001$ & $<0,001$ & $<0,001$ & $<0,001$ & $<0,001$ \\
\hline
\end{tabular}

$\mathrm{MP}_{2,5}$ : material particulado de 2,5 $\mu \mathrm{g} ; \mathrm{MP}_{10}$ : material particulado de $10 \mu \mathrm{g}$; CO: monóxido de carbono; $\mathrm{NO}_{2}$ : dióxido de nitrógeno; $\mathrm{O}_{3}$ : ozono; $\mathrm{SO}_{2}$ : dióxido de azufre; P: promedio de concentraciones de cada contaminante; D.S.: desviación estándar; V.C.: varianza conjunta. Concentraciones de $\mathrm{MP}_{2,5}$ y $\mathrm{MP}_{10}$ medidos en $\mu \mathrm{g} / \mathrm{m}^{3}$, de $\mathrm{CO}$ medido en ppm, de $\mathrm{NO}_{2}, \mathrm{O}_{3}$ y $\mathrm{SO}_{2}$ medidos en ppb. Cálculos realizados considerando 1.826 días en total (460 de invierno, 451 de verano) y 909 grados de libertad. Valor $p$ para dos colas. Fuente: Elaboración propia con datos obtenidos de la referencia ${ }^{21}$.

Tabla 2. Atenciones de urgencia en relación a los períodos de exposición a contaminantes (inviernos y veranos entre 2010 y 2014)

\begin{tabular}{|cccc|}
\hline Períodos de exposición & Respiratorias & $\begin{array}{c}\text { Atenciones de urgencia } \\
\text { Otra causa }\end{array}$ & Total \\
\hline Inviernos & 98.690 & 99.348 & 198.038 \\
Veranos & 49.330 & 112.459 & 161.789 \\
Total & 148.020 & 211.807 & 359.827 \\
\hline
\end{tabular}

Odds ratio 2,2646 (IC 95\% 2,2337-2,2960) a favor de relación invierno-enfermedades respiratorias y verano-otra causa; Odds ratio 0,4416 (IC 95\% 0,4355-0,4477) de la relación verano-enfermedades respiratorias e invierno-otra causa; $\chi^{2} 13.759,4947$ con un grado de libertad, valor $p<0,001$. Fuente: Elaboración propia con datos obtenidos de la referencia ${ }^{22}$.

Tabla 3. Coeficientes de correlación entre contaminantes

\begin{tabular}{|lcccccc|}
\hline & $\mathbf{M P}_{\mathbf{2 , 5}}$ & $\mathbf{M P}_{\mathbf{1 0}}$ & $\mathbf{C O}$ & $\mathbf{N O}_{\mathbf{2}}$ & $\mathbf{O}_{\mathbf{3}}$ & $\mathbf{S O}_{\mathbf{2}}$ \\
$\mathrm{MP}_{2,5}$ & 1,0000 & & & & \\
$\mathrm{MP}_{10}$ & 0,8175 & 1,0000 & & & \\
$\mathrm{CO}$ & 0,8509 & 0,6960 & 1,0000 & & & \\
$\mathrm{NO}_{2}$ & 0,7225 & 0,8100 & 0,7323 & 1,0000 & & \\
$\mathrm{O}_{3}$ & $-0,4945$ & $-0,2830$ & $-0,6439$ & $-0,5072$ & 1,0000 \\
$\mathrm{SO}_{2}$ & 0,3781 & 0,2981 & 0,4015 & 0,2989 & $-0,2122$ & 1,0000 \\
\hline
\end{tabular}

$\mathrm{MP}_{2,5}$ : material particulado de 2,5 $\mu \mathrm{g} ; \mathrm{MP}_{10}$ : material particulado de $10 \mu \mathrm{g}$; CO: monóxido de carbono; $\mathrm{NO}_{2}$ : dióxido de nitrógeno; $\mathrm{O}_{3}$ : ozono; $\mathrm{SO}_{2}$ : dióxido de azufre. Valores corresponden a coeficientes de correlación, prueba t-Student y valor $p<0,001$ para dos colas. Fuente: Elaboración propia con datos obtenidos de la referencia ${ }^{21}$. 
Tabla 4. Coeficientes de correlación entre atenciones de urgencia por enfermedades respiratorias y concentración de contaminantes del aire sin y con desfase de tiempo

\begin{tabular}{|lccccccccccc|}
\hline $\begin{array}{l}\text { Conta- } \\
\text { minante }\end{array}$ & $\mathbf{0}$ & $\mathbf{1 0}$ & $\mathbf{2 0}$ & $\mathbf{3 0}$ & $\mathbf{4 0}$ & $\mathbf{5 0}$ & $\mathbf{6 0}$ & $\mathbf{7 0}$ & $\mathbf{8 0}$ & $\mathbf{9 0}$ & $\mathbf{1 0 0}$ \\
$\mathrm{MP}_{2,5}$ & 0,3477 & 0,3505 & 0,3489 & 0,2933 & 0,3257 & 0,3141 & 0,2846 & 0,3083 & 0,3244 & 0,2654 & 0,1850 \\
$\mathrm{MP}_{10}$ & 0,2102 & 0,2382 & 0,2986 & 0,2287 & 0,2059 & 0,2684 & 0,3025 & 0,2282 & 0,2825 & 0,3001 & 0,2099 \\
$\mathrm{CO}$ & 0,4626 & 0,4429 & 0,4132 & 0,3847 & 0,3850 & 0,3346 & 0,2771 & 0,2974 & 0,2484 & 0,1759 & 0,1301 \\
$\mathrm{NO}_{2}$ & 0,3306 & 0,3956 & 0,4669 & 0,3835 & 0,2959 & 0,3578 & 0,3871 & 0,2266 & 0,2556 & 0,2701 & 0,1535 \\
$\mathrm{O}_{3}$ & $-0,3902$ & $-0,4591$ & $-0,4834$ & $-0,4394$ & $-0,3723$ & $-0,3781$ & $-0,3732$ & $-0,2453$ & $-0,2292$ & $-0,1985$ & $-0,1430$ \\
$\mathrm{SO}_{2}$ & 0,2105 & 0,1846 & 0,1541 & 0,1505 & 0,1228 & 0,1582 & 0,1368 & 0,1035 & 0,1166 & 0,0632 & 0,0413 \\
\hline
\end{tabular}

$\mathrm{MP}_{2,5}$ : material particulado de 2,5 $\mu \mathrm{g}$; $\mathrm{MP}_{10}$ : material particulado de $10 \mu \mathrm{g}$; CO: monóxido de carbono; $\mathrm{NO}_{2}$ : dióxido de nitrógeno; $\mathrm{O}_{3}$ : ozono; $\mathrm{SO}_{2}$ : dióxido de azufre. Valores corresponden a coeficientes de correlación, prueba estadística t-Student, valor $\mathrm{p}<0,001$ (excepto para $\mathrm{SO}_{2}$ con desfase de 90 y 100 días, con valores $p<0,01$ y $<0,1$, respectivamente) para dos colas. Fuente: Elaboración propia con datos obtenidos de las referencias ${ }^{21,22}$

- El CO tuvo la máxima correlación $(0,4626)$ con las enfermedades respiratorias alrededor del día 0 y luego tendió a decaer progresivamente sin nuevos peaks destacados.

- Las correlaciones del $\mathrm{MP}_{25}$ tuvieron tres máximos en los días 10, 40 y 80 con resultados $0,3505,0,3257$ y 0,3244 , respectivamente.

- Al comparar las enfermedades respiratorias 20, 60 y 90 días después de las mediciones del $\mathrm{MP}_{10}$ también hubo tres máximos $(0,2986,0,3025$ y 0,3001 , respectivamente).

- $\quad$ El SO tuvo su correlación máxima $(0,2105)$ alrededor del día 0 y posteriormente no hubo máximos destacables.

Para todos estos casos, el estadístico utilizado es t-Student y el valor $\mathrm{p}$ asociado es $<0,001$, excepto $\mathrm{SO}_{2}$ con desfase de 90 y 100 días (valores $\mathrm{p}<0,01 \mathrm{y}<0,1$, respectivamente).

\section{Discusión}

Este trabajo corrobora la asociación directa que existe entre las enfermedades respiratorias y la estación invernal, representado por la cantidad diaria de atenciones de urgencia en la comuna de El Bosque, con una odds ratio de 2,2646. Además, establece notorias diferencias entre la concentración diaria de material particulado y gases entre invierno y verano, con aumento del $\mathrm{MP}_{25}, \mathrm{MP}_{10}$, $\mathrm{CO}, \mathrm{NO}_{2}$ y $\mathrm{SO}_{2}$ en la estación fría y del $\mathrm{O}_{3}$ en la estación cálida.
Las mayores asociaciones entre contaminantes se dan entre $\mathrm{MP}_{2,5}, \mathrm{MP}_{10}, \mathrm{CO}$ y NO $\mathrm{N}_{2}$, con coeficientes de correlación mayores a 0,69 . $\mathrm{El} \mathrm{SO}_{2}$ tiene coeficientes de menor significancia con respecto al resto, mientras que el $\mathrm{O}_{3}$ presenta correlaciones negativas con respecto a todos los otros gases y material particulado.

La correlación más significativa entre atenciones de urgencia por enfermedades respiratorias y contaminantes del aire se ha dado con respecto al $\mathrm{O}_{3}$, cuyo resultado tiene signo negativo. Esto no significa que sea un factor protector, sino que posiblemente está representando el comportamiento de otra variable, la temperatura, porque la concentración del $\mathrm{O}_{3}$ aumenta en relación al incremento de temperatura, predominando ambos en verano. El coeficiente de correlación más cercano a -1 se da con una diferencia de 20 días, indicando que el efecto no es inmediato, sino que puede responder a los procesos propios de enfermedad y mejoría del sistema respiratorio.

El NO y el CO presentan correlaciones similares con respecto a las atenciones por enfermedades respiratorias. El primero, con un máximo a los 20 días y otro a los 60 días, lo que se puede interpretar como un efecto a corto y otro a mediano plazo. El segundo tiene su máximo en forma inmediata, disminuyendo progresivamente a medida que se desfasa el tiempo, lo que pudiera indicar que no hay consecuencias posteriores para la población, pero que niveles tóxicos serían potencialmente letales en forma más rápida. 
En la relación de atenciones por enfermedades respiratorias y material particulado, tanto el $\mathrm{MP}_{2,5}$ como el MP ${ }_{10}$ presentaron tres correlaciones máximas con valores no tan destacados como los casos anteriores, a pesar de sobrepasar los rangos recomendados por la Organización Mundial de la Salud.

$\mathrm{El} \mathrm{SO}_{2}$ es el que, según este estudio, tiene menos relación con las enfermedades respiratorias y, posiblemente, su importancia radica en la toxicidad que puede ocasionar en otros sistemas u órganos.

La motivación de este estudio es la necesidad que tiene cada zona afectada de contar con antecedentes de su propia realidad y no solamente con información importada de otros lugares, permitiendo la generación de soluciones adecuadas para ese entorno.

Aunque exista la imposibilidad de separar cada una de las variables implicadas en la contaminación del aire, se ha tratado de disminuir al máximo el sesgo, tomando como población de estudio aquella que vive y se atiende en la misma zona geográfica, y en la que es posible tener todas las mediciones deseadas en relación a contaminantes del aire. Lamentablemente, no se puede establecer cuántas horas al día la población en cuestión está dentro de la comuna o fuera de ella (por estudio, trabajo, etc.).

Agradecimientos: Este trabajo fue apoyado por Proyectos Basales y la Vicerrectoría de Investigación, Desarrollo e Innovación de la Universidad de Santiago de Chile. Chile.

\section{Referencias}

1. World Health Organization. WHO air quality guidelines for particulate matter, ozone, nitrogen dioxide and sulfur dioxide: Global update 2005 [Internet]. Ginebra: WHO Press; 2006 [Citado el 19 de octubre de 2016]. Disponible en: http://www.who.int/phe/health_topics/ outdoorair/outdoorair_aqg/en/

2. Ostro B, Sánchez J, Aranda C, Eskeland G. Air pollution and mortality: Results from Santiago, Chile. Policy Research Working Paper 1453. Washington D.C.: World Bank, Policy Research Department (Public Economics Division); 1995 Mayo.

3. Hong Y, Leem J, Ha E, Christiani D. PM(10) exposure, gaseous pollutants, and daily mortality in Inchon, South Korea. Environ Health Perspect 1999; 107 (11): 873-8. Disponible en: https://www.ncbi.nlm.nih.gov/pmc/ journals/253/ [Consultado el 19 de octubre de 2016].

4. Fisher P, Marra M, Ameling C, Hoek G, Beelen R, de Hoogh K, et al. Air pollution and mortality in seven million adults: The Dutch Environmental Longitudinal Study (DUELS). Environ Health Perspect 2015; 123 (7): 697-704. Disponible en: http://doi.org/10.1289/ ehp.1408254 [Consultado el 19 de octubre de 2016].

5. Zhang Z, Laden F, Forman J, Hart J. Long-term exposure to particulate matter and self-reported hypertension: A prospective analysis in the Nurses' Health Study. Environ Health Perspect 2016; 124 (9): 1414-20. Disponible en: http://doi.org/10.1289/EHP163 [Consultado el 19 de octubre de 2016].

6. Chen H, Burnett R, Copes R, Kwong J, Villeneuve P, Goldberg M, et al. Ambient fine particulate matter and mortality among survivors of myocardial infarction: Population-based cohort study. Environ Health Perspect 2016; 124 (9): 1421-8. Disponible en: http://doi. org/10.1289/EHP185 [Consultado el 19 de octubre de 2016].

7. Aaron C, Chervona Y, Kawut S, Diez-Roux A, Shen $\mathrm{M}$, Bluemke D, et al. Particulate matter exposure and cardiopulmonary differences in the Multi-Ethnic Study of Atherosclerosis. Environ Health Perspect 2016; 124 (8): 1166-73. Disponible en: http://doi.org/10.1289/ ehp.1409451 [Consultado el 19 de octubre de 2016].

8. Gehring U, Gruzieva O, Agius R, Beelen R, Custovic A, Cyrys J, et al. Air pollution exposure and lung function in children: The ESCAPE Project. Environ Health Perspect 2013; 121 (11-12): 1357-64. Disponible en: http:// doi.org/10.1289/ehp.1306770.

9. Tétreault L, Doucet M, Gamache P, Fournier M, Brand A, Kosatsky T, et al. Childhood exposure to ambient air pollutants and the onset of asthma: An administrative cohort study in Québec. Environ Health Perspect 2016; 124 (8): 1276-82. Disponible en: http://doi.org/10.1289/ ehp. 1509838.

10. Mehta A, Zanobetti A, Bind M, Kloog I, Koutrakis P, Sparrow D, et al. Long-term exposure to ambient fine particulate matter and renal function in older men: The Veterans Administration Normative Aging Study. Environ Health Perspect 2016; 124 (9): 1353-60. Disponible en: http://doi.org/10.1289/ehp.1510269.

11. Tzivian L, Dlugaj M, Winkler A, Weinmayr G, Hennig F, Fuks KB. Long-term air pollution and traffic noise exposures and mild cognitive impairment in older adults: A cross-sectional analysis of the Heinz Nixdorf Recall Study. Environ Health Perspect 2016; 124 (9): 1361-8. Disponible en: http://doi.org/10.1289/ehp.1509824.

12. Sistema de Información Nacional de Calidad del Aire del Ministerio del Medio Ambiente de Chile [Internet]; 
c2009-2015 [Consultado el 16 de octubre de 2016]. SINCA: Sistema de Información Nacional de Calidad del Aire; [alrededor de 3 pantallas]. Disponible en: http:// sinca.mma.gob.cl/index.php/, http://sinca.mma.gob.cl/ index.php/pagina/index/id/norma y http://sinca.mma. gob.cl/index.php/redes

13. Zamorano A, Márquez S, Aránguiz J, Bedregal P, Sánchez I. Relación entre bronquiolitis aguda con factores climáticos y contaminación ambiental. Rev Med Chile 2003; 131: 1117-22.

14. Romero H, Irarrázaval F, Opazo D, Salgado M, Smith P. Climas urbanos y contaminación atmosférica en Santiago de Chile. Eure 2010; 36 (109): 35-62.

15. Avendaño L, Céspedes A, Stecher X, Palomino M. Influencia de virus respiratorios, frío y contaminación aérea en la infección respiratoria aguda baja del lactante. Rev Med Chile 1999; 127 (9): 1073-78.

16. Cerda A, García L. Contaminación del aire en la Florida (Talca, Chile): Beneficios económicos en salud por la reducción de los niveles PM10. Rev Med Chile 2010; 138: 1395-402.

17. Sarnat J, Schwartz J, Catalano P, Suh H. Gaseous pollutants in particulate matter epidemiology: confounders or surrogates? Environ Health Perspect 2001; 109 (10): 1053-61. Disponible en: https://www.ncbi.nlm.nih.gov/ pmc/articles/PMC1242083/

18. Schwartz J. Is the association of airborne particles with daily deaths confounded by gaseous air pollutants? An approach to control by matching. Environ Health Perspect 2004; 112 (5): 557-61. Disponible en: https://www. ncbi.nlm.nih.gov/pmc/articles/PMC1241921/

19. Biblioteca del Congreso Nacional de Chile. Reporte estadístico comunal 2015: El Bosque [Internet]. 2015 [Consultado el 16 de octubre de 2016]. Disponible en: http://reportescomunales.bcn.cl/2015/PDF/El_Bosque. pdf.
20. Dirección de Salud El Bosque de la Ilustre Municipalidad de El Bosque [Internet]. c2015 [Consultado el 08 de enero de 2017]. Centros de salud; [alrededor de 11 pantallas]. Disponible en: http://www.saludelbosque.cl/index.php/centros-de-salud/cesfam-condores-de-chile.

21. Sistema de Información Nacional de Calidad del Aire del Ministerio del Medio Ambiente de Chile [Internet]. Estaciones de Monitoreo de la Calidad del Aire: Región Metropolitana de Santiago. c2015 [Actualizado el 05 de enero de 2017; Consultado el 08 de enero de 2017]. Disponible en: http://sinca.mma.gob.cl/index.php/ region?id=M\&cache=off\& y http://sinca.mma.gob.cl/ index.php/region/index/id/M.

22. Departamento de Estadísticas e Información de Salud del Ministerio de Salud de Chile [Internet]. Reportes Minsal: Atenciones de Urgencia-Vista Diaria. [Actualizado el 05 de enero de 2017; Consultado el 08 de enero de 2017]. Disponible en: http://cognos.deis. cl/ibmcognos/cgi-bin/cognos.cgi?b_action=cognosViewer\&ui.action $=$ run\&ui.object $=\% 2$ Fcontent $\%$ 2Ffolder\%5B\%40name\%3D\%27PUB\%27\%5D\%2Ffolder\%5B\%40name\%3D\%27REPORTES\%27\%5D\%2Ffolder\%5B\%40name\%3D\%27Atenciones\%20de\%20 Urgencia $\% 27 \% 5 \mathrm{D} \% 2$ Freport $\% 5 B \% 40$ name $\% 3 \mathrm{D} \% 27 \mathrm{~A}$ tenciones $\% 20$ Urgencia $\% 20-\% 20$ Vista $\% 20$ diaria $\% 20$ -\%20Servicios $\% 27 \% 5$ D\&ui.name=Atenciones $\% 20 U r-$ gencia\%20-\%20Vista\%20diaria\%20-\%20Servicios\&run. outputFormat=\&run.prompt $=$ true

23. Medina F, Galván M. División de Estadística y Proyecciones Económicas, CEPAL, Naciones Unidas. Serie 54 Estudios estadísticos prospectivos-Imputación de datos: Teoría y práctica [Internet]. Santiago de Chile: CEPAL; 2007 [Citado el 19 de octubre de 2016]. Disponible en: http://www.cepal.org/es/publicaciones/4755-imputacion-datos-teoria-practica. 